\title{
Die Zeit für eine Aktualisierung der Leitlinien ist gekommen
}

Fragestellung: Im Rahmen einer Metaanalyse sollte die Frage beantwortet werden, welche oralen und topischen Medikamente in der Behandlung neuropathischer Schmerzen wirksam sind.

Hintergrund: In den letzten zehn Jahren sind zahlreiche Handlungsempfehlung zur Behandlung neuropathischer Schmerzen oder Schmerzsyndrome gegeben worden. Zwischenzeitlich ist jedoch die Zahl der dazu publizierten Daten und noch laufenden klinischen Studien deutlich angestiegen, sodass eine Aktualisierung dieser Empfehlungen sinnvoll ist, auch vor dem Hintergrund, dass durch die Einführung des GRADE-Systems eine genauere Messung des Evidenzgrades der vorliegenden Daten und Empfehlungen möglich ist.

Finnerup NB, Attal N, Haroutounian $\mathrm{S}$ et al. Pharmacotherapy of neuropathic pain in adults: a systematic review and metaanalysis. Lancet Neurol 2015; 14: $129-30$
Patienten und Methodik: Für diese Metaanalyse und systematischen Review wurden 229 randomisierte doppelblinde placebokontrolierte Studien, in peer-reviewed Journals und unpublizierte Studien von 1966 bis heute eingeschlossen. Als primäre Variable wurde die Number-needed to-treat (NNT) für eine mindestens 50 \%ige Schmerzreduktion definiert.

Ergebnisse: Die Analyse des Publikationsbias wurde mit $10 \%$ geschätzt. Für die Verwendung von SNRI (z. B. Duloxetin) wurde eine NNT von 6,4 berechnet, 7,7 für Pregabalin, 7,2 für Gabapentin und 10,6 für topisches Capsaicin. Für Trizyklika, Opioide und lokales Lidocain waren die NNT niedriger. Die Verträglichkeit und Sicherheit war bei topischen Substanzen höher, die Kosten für Trizyklika und Tramadol waren deutlich niedriger.

Schlussfolgerungen: Basierend auf dieser Metaanalyse empfehlen die Autoren die Verwendung von Trizyklika, SNRI, Pregabalin und Gabapentin als First-line-Medikamente. Die Verwendung von Lidocain- und Capsaicin-Pflaster sowie Tramadol werden als Second-line-Option empfohlen, während für die Verwendung von hochpotenten Opioiden und Botulinumtoxin A lediglich eine schwache Empfehlung gegeben wird. Die Autoren raten auf der Basis ihrer Daten zu einer Aktualisierung der bisherigen Leitlinien zur Behandlung neuropathischer Schmerzen.

\section{- Kommentar von Tim Hagenacker, Essen}

\section{Im Detail zeigen sich durchaus Änderungen}

Diese Metaanalyse unterstreicht, dass eine Aktualisierung der Leitlinien zur Behandlung neuropathischer Schmerzen nun vorgenommen werden sollte. Die Analyse stützt sich dabei vornehmlich auf den wesentlichsten Parameter der Therapie, nämlich die Schmerzreduktion, ohne die Sicherheit und Verträglichkeit und letztlich auch ökonomischen Parameter zur vernachlässigen. Wenngleich sich aus dieser Metaanalyse zwar keine bahnbrechenden Änderungen der bisherigen medikamentösen Behandlung neuropathischer Schmerzen ableiten lassen, ist eine Überarbeitung der Leitlinien nichtsdestotrotz sinnvoll, weil sich im Detail doch durchaus Änderungen zei-

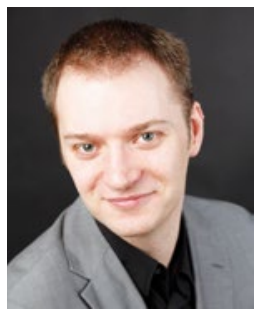

PD Dr. med. Tim Hagenacker, Essen

Klinik für Neurologie,

Universitätsklinikum Essen

E-Mail: tim.hagenacker@uk-essen.de gen, die für die Behandlung des einzelnen Patienten in der Praxis wichtig sind. Der einzige Stolperstein an dieser Metaanalyse ist, dass alle Entitäten, die sich hinter neuropathischen Schmerzen verbergen, in einer Analyse vereinheitlicht werden, wenngleich sich die pathophysiologischen Mechanismen und die daraus abzuleitenden Therapiemaßnahmen unterscheiden können. Dieser Punkt wird aber weiterhin diskutiert und muss sicherlich im Rahmen eines Updates der Leitlinien berücksichtigt werden.

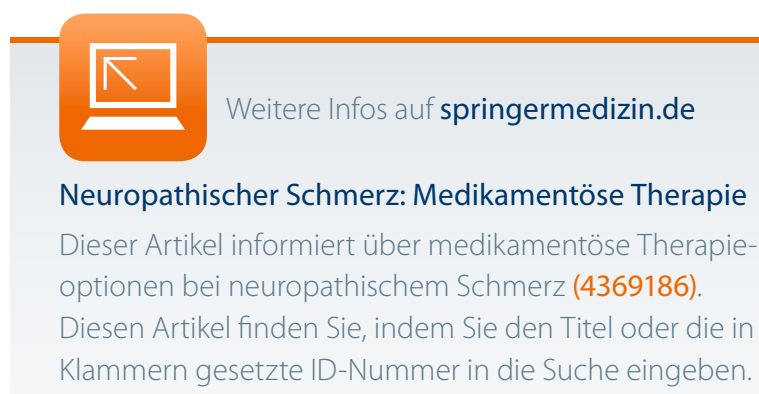

